\title{
Three Thresholds for the Efficiency in Energy Management in WSN
}

\author{
Yacouba Ouattara, Christophe Lang, and Adel Elgaber
}

\begin{abstract}
Nowadays, the domain of sensor networks constitutes a very active research field. People are using a lot of low cost networks. As a result, they are facing an important energy management problem. Consequently, Researchers have tried to ensure long life to networks [1]-[8]. LEACH, PEGASUS, TEEN and APTEEN are pioneers in the reduction of WSN energy consumption. Generally, cluster heads $(\mathrm{CH})$ and nodes close to the station die quickly in sensor networks. Cluster heads consume more than simple nodes. Also, the needed energy for the formation of clusters is very high in sensor networks.

We propose an algorithm that aims at reducing the energy consumption in the formation of clusters and at providing a long life to the homogeneous sensor networks. In this paper, we present this algorithm for energy management in sensor networks. It is based on three thresholds. What we propose is a new way to construct clusters. It will allow having a real vision on the energy available in the sensor network at anytime. Our experimentations have shown a reduction in the energy used in comparaison with other methods.
\end{abstract}

Index Terms-Wirless, WSN, routing.

\section{INTRODUCTION}

In hierarchical clusters, our method provides a simple solution to save energy consumed in the choice of CHs. It also permits to save energy during the demand for information on the energy situation of other sensors.

The sensor failure can have serious consequences on the life and activities of the network. In the life of the network, it is necessary to involve nodes that have an energy capacity greater than a certain threshold to avoid catches and unnecessary presence. This will permit to avoid long transmission time. This, in return, will help to avoid losses and retransmission of messages.

Our solution allows proposing a maintenance algorithm to avoid a loss of energy caused by a failed node. It also saves the energy expended by the formation of clusters and cluster heads. We call our method the hybrid method.

\section{The Basics of LEACH Clustering}

Routing protocols are of two types. We have flat and hierarchical types. We are interested in hierarchical routing in a sensor network whose foundation bases are: LEACH, TEEN [9], APTEEN [10] and PEGASIS [11]. LEACH (Low Energy Adaptive Clustering Hierarchy) was introduced [3] in 2000. LEACH is considered as the founding father of clustering. This algorithm randomly selects 5 percent cluster

Manuscript received July 14, 2014; revised December 13, 2014.

Yacouba Ouattara, Christophe Lang and Adel Elgaber are with Université de Franche Comté, France (e-mail: yacouattt@yahoo.fr, christophe.lang@univ-fcomte.fr, aelgaber@femto-st.fr). head $(\mathrm{CH})$ nodes as homogeneous and offers a rotation of the role of $\mathrm{CH}$. The $\mathrm{CH}$ aggregate data are sent by other nodes in a simple message for direct transmission to the base station or through other $\mathrm{CHs}$. The $\mathrm{CH}$ can communicate with each other and also with the base station which is often a remote computer connected to the Internet. The $\mathrm{CHs}$ create a table to allocate a TDMA transmission time to each cluster member. Since each node knows in advance the time slot it will occupy, TDMA puts some nodes in a stand by situation. TDMA and the table created for each cluster will be broadcast to al cluster members. LEACH reduces energy consumption in sensor networks. When there are many nodes at a large scale, there is the problem of the choice of the well which uses the following probabilistic:

$$
T(i)=\left\{\begin{array}{l}
\frac{P}{1-P^{*}\left(r \bmod \frac{1}{P}\right)} \text { if } \mathrm{i} \text { was not already elected as a pivot } \\
0
\end{array}\right.
$$

$P$ is a ratio of $\mathrm{CH} ; r$ in $[0,1]$. If $r$ is smaller than the threshold value, $T(i)$, the node becomes a $\mathrm{CH}$ for the current cycle. The particularity of LEACH lies in the non-necessity of communication for the election of CHs. LEACH manages to save energy but the problem of widespread transmission arises.

Algorithms derived: From LEACH presented above, researchers have studied the mode of formation of clusters which consumes energy sensors. A mode based on the probabilistic option, the option of remaining energy nodes, the distance between nodes or a combination can elect the $\mathrm{CH}$ and cluster formation. - EECS (An Energy Efficient Clustering Scheme in Wireless Sensor Networks), published in 2005, [12] deals with the periodic collection by forming clusters and CHs locally formed and based on the energy of sensors.

Beyond LEACH, the authors rely on a load balancing between the $\mathrm{CH}$. The problem remains widespread. - MHEED (Multihop HEED) Unlike HEED, [13] introduced two variants.

The first variant is based on using the best path built to convey data. In the second, the issuer chooses its next relay probabilistic way to balance the load between different relays. MHEED attempts to solve the problem of scale in HEED. The probabilistic approach leads to the formation of unequally sized clusters, which leads to imbalance in energy consumption across the network and thereby reduces the efficiency and network lifetime. 
A second mode is the involvement of the base station in the choice of $\mathrm{CH}$ and clusters. Communication between nodes for the appointment of the head cluster is a source of energy consumption. To solve this problem, we turn to the base station to allow the setting up of cluster heads. Thus, we have - LE2AC (Low Energy Adaptive Connectionist Clustering). Like the above algorithms, [14] and [15] are based on the base station to establish its clusters. The nodes are equipped with GPS that allows the node to communicate its position and amount of energy. The base station will use the method in the Kohonen artificial neural network based methods of unsupervised learning to construct its clusters and elect the $\mathrm{CH}$ based on the geographical location and energy.

All nodes communicate early with the base station which is huge in consumption and also in large scale; all nodes cannot reach the base station. - EEUC (An Energy-Efficient Distributed Unequal Clustering Protocol for Wireless Sensor Networks) EEUC is proposed in [16] to solve the problem of nodes that are close to the base station and are used extensively. It offers an uneven distribution of clusters with a high percentage of clusters away from the base station and a small percentage of clusters close to the base station.

In this article, we focus on the probabilistic algorithm HEED. This is the most used in the literature.

\section{HEED (A Hybrid, Energy-Efficient, Distributed)}

In 2004, Ossama Younis and Sonia Fahmy HEED wrote [17]. They introduced a cost function for each sensor which is sent to each neighbor. HEED is selected with the $\mathrm{CH}$ whose cost function is minimal. Iterations with a probabilistic method are also used for the self-election of $\mathrm{CHs}$, but with consideration of the residual energy of each sensor. The formula is:

$$
C H_{\text {prob }}=\max \left(C_{\text {prob }} \frac{E_{i}}{E_{\max }}, P_{\min }\right)
$$

$C_{\text {prob }}$ is the desired ratio of $\mathrm{CHs}$ on the network, $E_{i}$ the residual energy of sensor $\mathrm{i}$ and $P$ min is a minimum probability eligibility to ensure the termination of the algorithm (to avoid an infinite number of iterations). At each iteration $C_{\text {prob }}$ is multiplied by 2 (to a maximum of 1 when the sensor is guaranteed to be elected $\mathrm{CH}$ ).

Thus, HEED uses waste heat at the choice of $\mathrm{CHs}$ and inter cluster communication cost for nodes that are between two clusters. But, it does not resolve the extent of a large scale.

Solutions above are able to save energy, to give a long life to the sensor network [2], [18]. But they have their limits [19], [20], [21], for example the ability of a node to self-evaluation in order to provide a longer life to the network. Our solutions will provide an alternative for a long lifetime of the sensor network.

\section{OUR AlgORITHM BASED ON THREE THRESHOLDS}

In hierarchical clusters, our method provides a simple solution to save energy consumed in the choice of CHs. It also permits to save energy during the demand for information on the energy situation of other sensors. Our solution will use the base station to create zones and the original CHS. Then, the base station will leave the choice of the next $\mathrm{CHs}$ to the original one. Then, it goes on. The sensor failure can have serious consequences on the life and activities of the network. In the life of the network, it is necessary to involve nodes that have an energy capacity greater than a certain threshold to avoid catches and unnecessary presence. This will permit to avoid long transmission time. This, in return, will help to avoid losses and retransmission of messages. Our solution allows proposing a maintenance algorithm to avoid a loss of energy caused by a failed node. It also saves the energy expended by the formation of clusters and cluster heads. We call our method the hybrid method.

\section{A. Principle}

The position of the base station is included in the energy consumption of the sensors. The establishment of clusters is also very difficult to manage sensor network. It is the same for the appointment of heads of clusters. It consumes a lot of energy. During the operation of the networks, the nodes slowly deplete their energy. This energy reaches a level where the node is no longer effective and therefore harmful to the operation of the network. These nodes can capture data but cannot send. They warn neighboring nodes to remain awake to receive data. To cope with the huge energy losses, our following solution is to give a long life to the network, to conserve energy so as to give a long life to the network. A simple solution is to divide symmetrically several ground areas compared to the size of the field and consider a percentage of ten percent of each zone as cluster head. The symmetrical division of land into several zones eliminates unnecessary nodes in the network operation by providing thresholds for each cluster and each zone.

\section{B. Description of the Algorithm}

The construction of our network is using the base station.

The base station divides the field into several zones. Then, it designates area $\mathrm{CHs}$ among nearby nodes. (step1) To do this, the first idea is to compute a minimum energy and maximum energy consumption for each cluster. For this purpose, the minimum distance is derived from the node closest to the $\mathrm{CH}$ and the maximum distance is derived from the farthest node from the $\mathrm{CH}$. We use the formulas of energy for this idea. This idea allows nodes to assess themselves in terms of energy stability and long lifetime of the network. These calculations are carried out at each $\mathrm{CH}$ and are communicated to the nodes for self-assessment (step2). The second idea is that our method permits the $\mathrm{CH}$ to assess itself by receiving energy from the nodes. When a node needs to send data to Cluster Head $(\mathrm{CH})$, it must ensure its own energy capacity to prevent data loss and network stability. CHs take into account only the nodes that participate in the cluster and send data to destination. It takes into account only the nodes that are alive and can provide information (step3). The third is to enable heuristic $\mathrm{CHs}$ to determine their successors in the function of averaging (step4). It will designate the new $\mathrm{CHs}$ which are next to the old $\mathrm{CHs}$ with energy that is higher than the average. To sum up, there are the five following points.

1) Division of the field into several zones and the designation of cluster heads

2) Determination of the minimum and maximum consumption of each cluster

3) Sending parameters to other nodes in the cluster for 


\section{self-assessment}

4) Sending a message to $\mathrm{CH}$ involves the capture of the message and the amount of energy of each node that will be used by the $\mathrm{CH}$ to rebuild the cluster

In the following, we describe formulas and algorithms that are used in this method.

1) Construct zones and the cluster (step 1): In this part, we present the algorithm used to determine the division of the field into several zones and to construct the network with its cluster heads. Probabilistic algorithms consume a lot of energy in the communication because of the choice of CHs. On average, we can estimate $n *(n-1)$ consumption of energy. Considering $n$ node, there will be broadcast transmissions where there are $\mathrm{n}$ and $\mathrm{n}-1$ operations. This is huge for the establishment of the network. The zoning by the base station reduces the number of communications and $\mathrm{CH}$ nodes. This saves energy by limiting the communication of the sensors to their areas. Dividing the field into several zones with respect to the size of the field (symmetric sharing) or with respect to the concentration of the nodes:

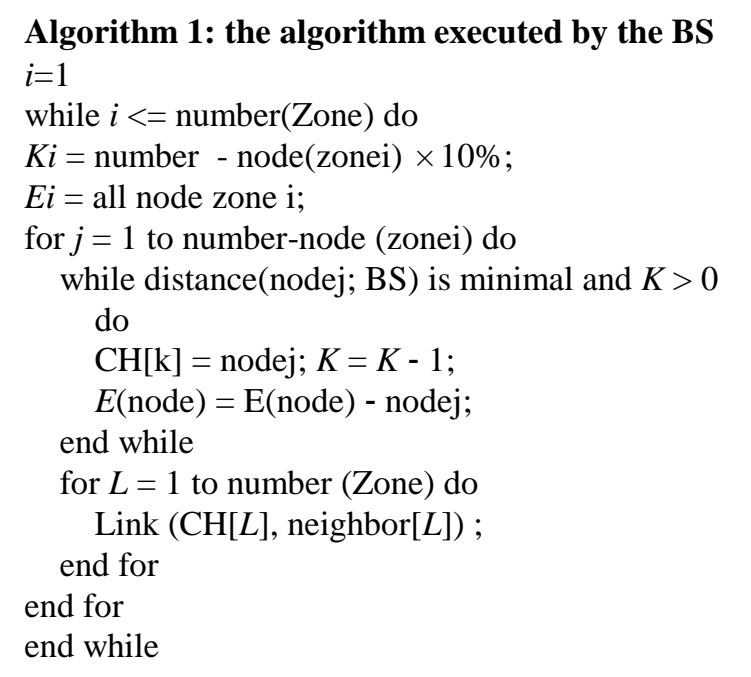

2) Determination of thresholds of each cluster (step 2):

In this section, we try to determine levels of thresholds that are necessary in our method. For this, we need some formulas taken from the literature.

Ref. [6] gives the formulas compared to a message of $\mathrm{k}$ bits of a distance of meters.

The transmitter consumes:

$\operatorname{Et}(k ; d)=\operatorname{Eelec}(k)+\operatorname{Eamp}(k ; d)=\operatorname{Eelec} \times k+\operatorname{Eamp} \times k \times d^{2}$

And the receiver consumes:

$$
\operatorname{Er}(k)=\operatorname{Eelec}(k)=\operatorname{Eelec} \times k
$$

Adding these two formulas, we deduce that we need a quantity:

$$
\operatorname{ERT}(k ; d)=2 \times \text { Eelec } \times k+\text { Eamp } \times k \times d^{2}
$$

To cope with these huge energy losses, our algorithm determines the threshold below which the sensor turns off.
At one point of the operation, each sensor must assess itself to avoid an unhealthy use of energy and that of others. They alert neighboring nodes which remained awake to receive data.

3) Nodes Behavior (step 3): During the network lifetime, nodes follow a particular algorithm. This one determines if the node is able to stay alive in the network.

\section{Algorithm 2: the algorithm executed by the $\mathbf{C H}$}

Determines the distance from the nearest node (Nmin) of $\mathrm{CH}$. Determines the distance of the farthest node (Nmax) of $\mathrm{CH}$ Emin (threshold 1) and Emax (threshold 2) are calculated from 1 and 2

$$
\begin{gathered}
E_{\min }(k ; d)=2 \times E_{\text {elec }} \times k+E_{\mathrm{amp}} \times k \times N \min \\
E_{\max }(k ; d)=2 \times E_{\text {elec }} \times k+E_{\mathrm{amp}} \times k \times N \max ^{2} \\
\text { send }\left(E_{\min } ; E_{\max } ; \text { nodes }\right)
\end{gathered}
$$

\section{Algorithm 3: the algorithm executed by the node}

$E_{i}$ is the energy of the node $\mathrm{i}$

If $E_{i}<$ Emin then turn off the node

else

if $\left(E_{i}>=E_{\max }\right)$ then send the message to the $\mathrm{CH}$

else endif

Sending the message to the node nearest endif

4) Threshold designation of the cluster head (step 4): We propose thresholds so as to give long life to the network by removing nodes that may affect the operation of the network.

It performs a data compression at each node.

In addition to the messages to capture and send, each node is added to the message or its quantity power capacity to allow the $\mathrm{CH}$ or the base station to implement an effective strategy for the establishment of a new cluster.

The $\mathrm{CH}$ can calculate the average energy. If the energy of $\mathrm{CH}$ is less than the average result, the $\mathrm{CH}$ triggers a new cluster formation and loses its role of $\mathrm{CH}$.

$$
\mathrm{CH}=\mathrm{P} 1 \_i \_n(E i) n
$$

The algorithm is iterative. At each iteration, the $\mathrm{CHs}$ calculate after each transmission to the base station.

We can transmit the succession role of a $\mathrm{CH}$ node by using the following criteria:

- The $\mathrm{CH}$ is the node with the maximum amount of energy in the cluster.

- The $\mathrm{CH}$ is the node closest to the center of gravity of the cluster (in the sense of physical distance).

- The $\mathrm{CH}$ is the node closest to the base station.

\section{EXPERIMENTATION}

In this part, we present some experimentation in order to evaluate our method. We compare it with well-known algorithms. In our simulation of the algorithm, we also 
simulated clustering algorithm LEACH and HEED using the SMAC platform (Simulation platform developed in Femto st laboratory).

\section{A. First Experimentations to Determine Initial Parameters}

The sensors which are randomly deployed in the field can be divided into several zones.

We observe through the Fig. 1-Fig. 4, that the number of zones or cluster has an impact on network performance.
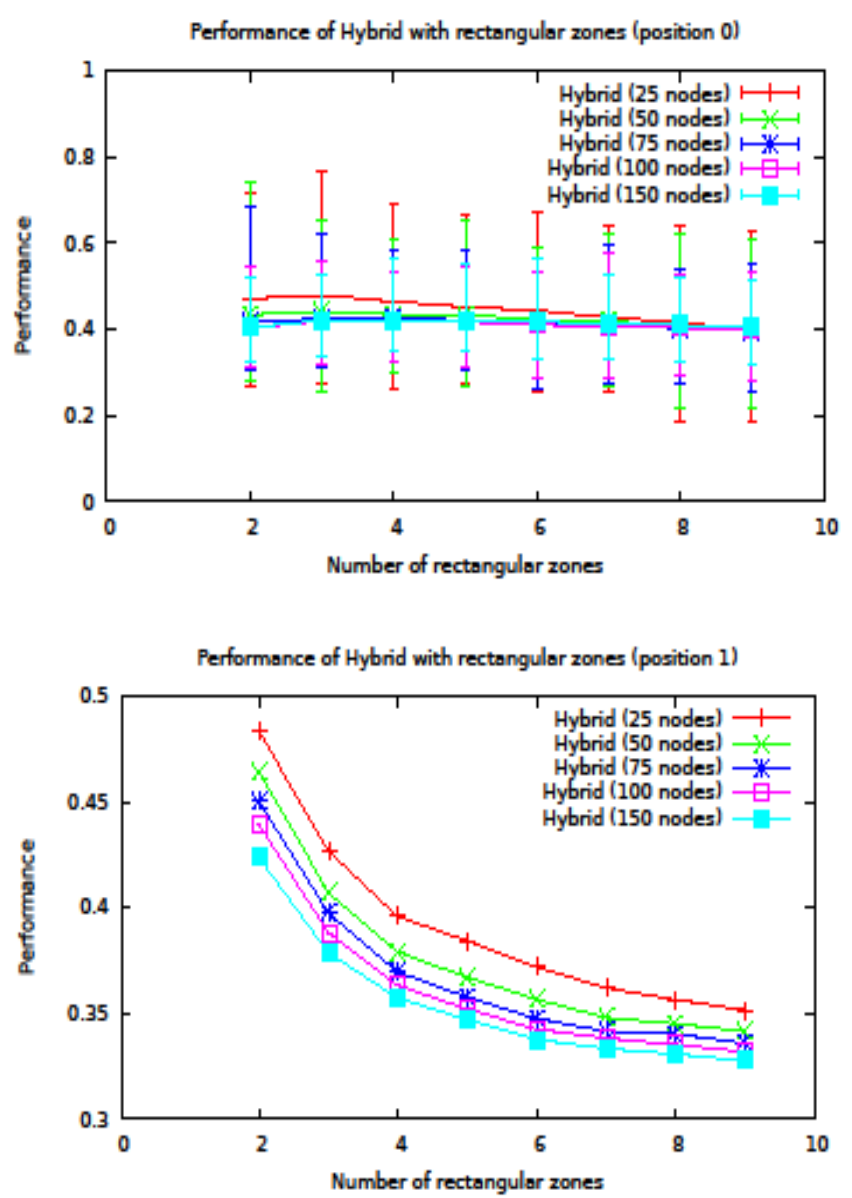

Fig. 1. Performance of hybrid with rectangular zones.

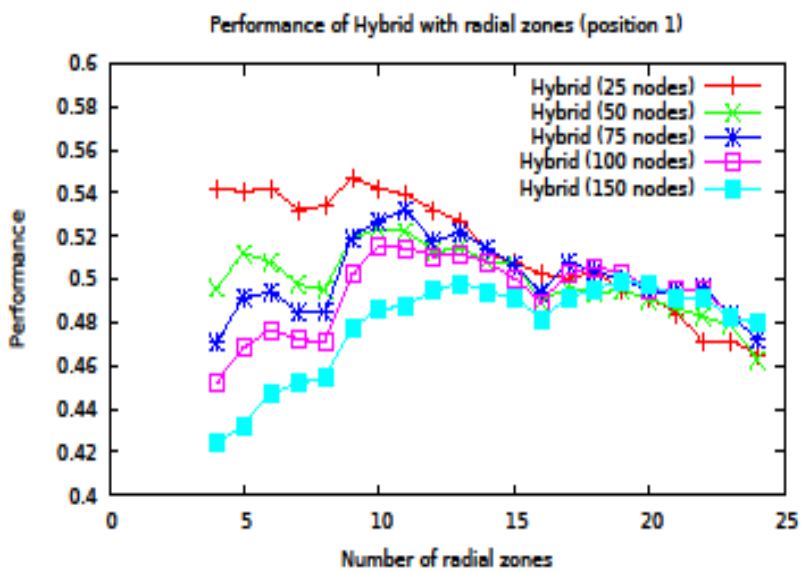

Fig. 2. Performance of hybrid with rectangular zones.

Moreover, the more there are areas, the greater the performance decreases. With different positions of the base station, see Fig. 6, we found that for the radial, the best performance is obtained with eight areas. For the rectangular form, the best performance is obtained with three zones. This section allows to solve the complex problem of clustering parameters. In the remainder of the experiment, the two numbers of the most successful areas are used.

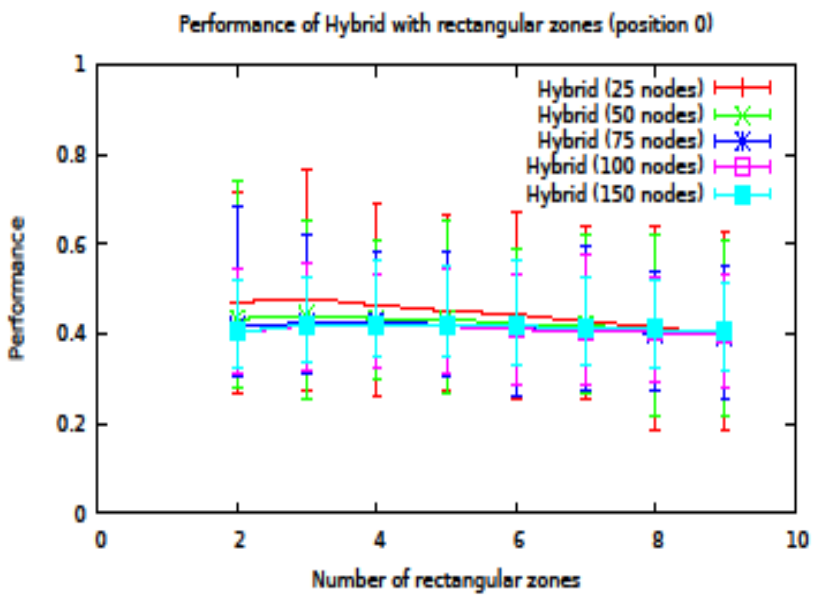

Fig. 3. Performance of hybrid with radial zones.

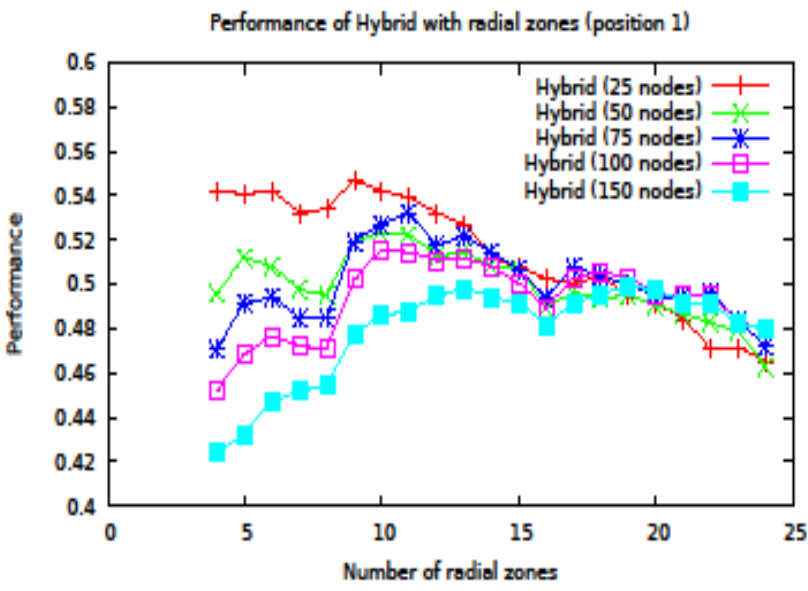

Fig. 4. Performance of hybrid with radial zones.

Let us compare our approach with methods LEACH, HEED.

We use the same communication model and the same data. The simulation parameters are in the Fig. 5.

\begin{tabular}{|l|c}
\hline Notation & Description \\
\hline $\mathrm{N}=[100,200,300,400]$ & numbers of nodes \\
$E_{o}=0.5$ joule & Energy initial of node \\
$E_{\text {elec }}=50$ nano-joule $/ \mathrm{bit}$ & Per bit energy consumption \\
$E_{a m p}=100$ peta-joule/bit/m2 & Amplifier transmitting energy \\
$E_{A}=5$ nano-joule / bit & Energy for data aggregation \\
$\mathrm{K}=2000$ bits & Message Size \\
Area & 400 X 400 \\
Position of the base station & P0 or P1 \\
\hline
\end{tabular}

Fig. 5. Experiment parameters.

At the beginning, we randomly deploy nodes in a given space $(400 \mathrm{mX} 400 \mathrm{~m})$, with the same amount of energy $(E=2 J)$. In our experimentation, we had a choice between rectangular and radial forms. We present the performance of our hybrid algorithm by setting the base station to the point $\mathrm{P} 0$ or $\mathrm{P} 1$.

Using the theorem distance calculation, we calculate an initial clustering of n4odes on the variables $x, y$ representing the coordinates of nodes by assigning roles to each node (or simply $\mathrm{CH}$ ). The energy consumption of each node is 
calculated by using the formulas cited using $k=2000$ bytes, Eelec $=50 \mathrm{~nJ} /$ bit and Eamp $=0.0013 \mathrm{pJ} / \mathrm{bit} / \mathrm{m} 2$ after each transmission.

The operation is repeated on the outcome of the previous step until there is the loss of a node or a $\mathrm{CH}$ due to lack of energy.

The $\mathrm{CHs}$ are selected in all the clusters according to a selection criteria listed above.

We considered that the measure network performance in our simulation is the number of messages sent to the base station over a lifetime, which is the time interval from the beginning of the operation of the sensor network until the death of the first node in the network. The position of the base station included in the energy consumption of the sensors (The graph of Fig. 6 clearly shows how the reliability of the average on the play position of the energy consumption). First, we placed the base station at P0 $(x=200, y=0)$ as shown in Fig. 6 . Secondly, we placed the base station at P1 $(x=400, y=0)$ as shown in Fig. 6. The BS at this position will have a similar connectivity as in the first scenario.

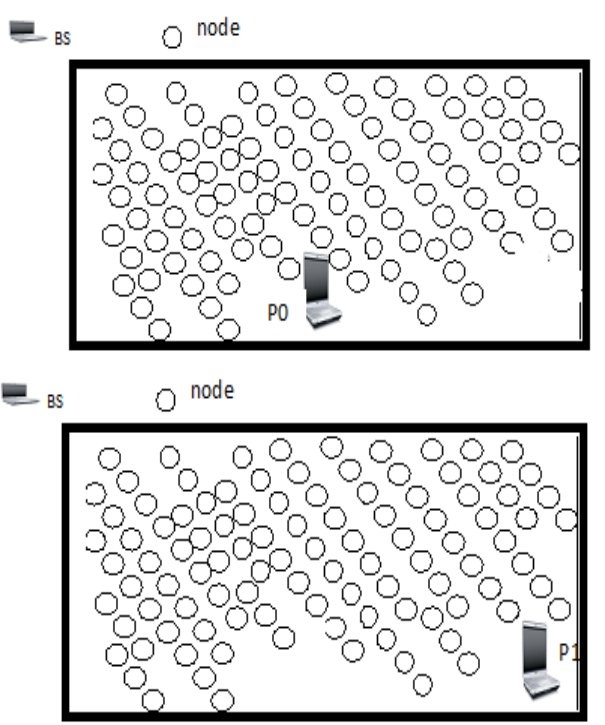

Fig. 6. Illustration of the placements of the base station.

\section{B. Comparison with the Other Methods}

LEACH, HEED and our algorithm give the following diagrams (Fig. 7 and Fig. 8). These figures show the performance of our hybrid algorithm compared with LEACH and HEED.

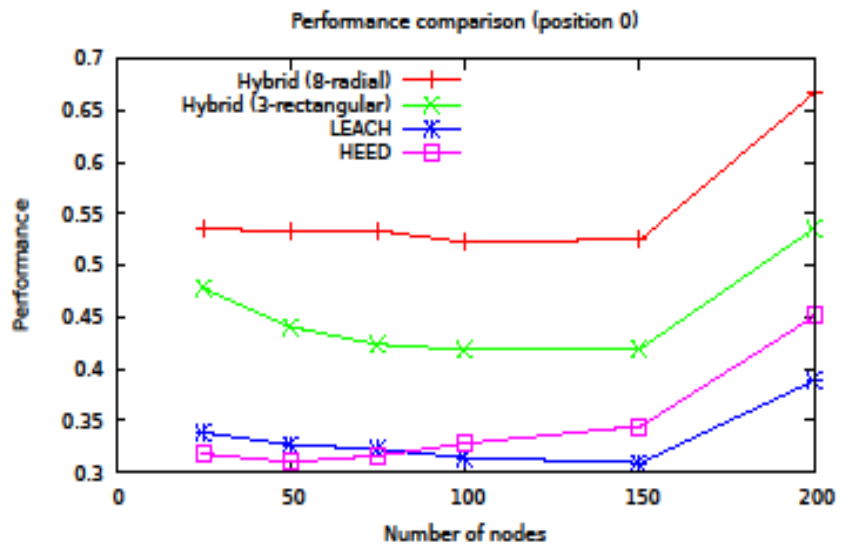

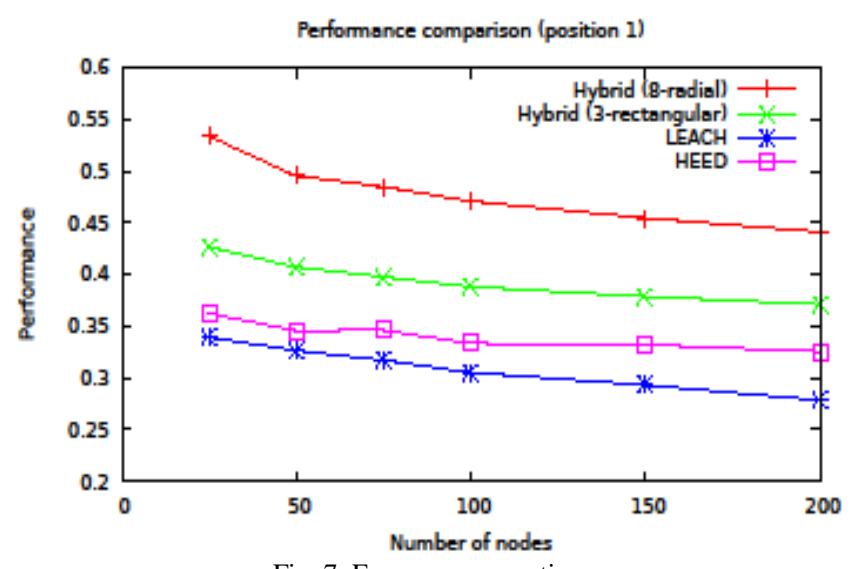

Fig. 7. Energy consumption.

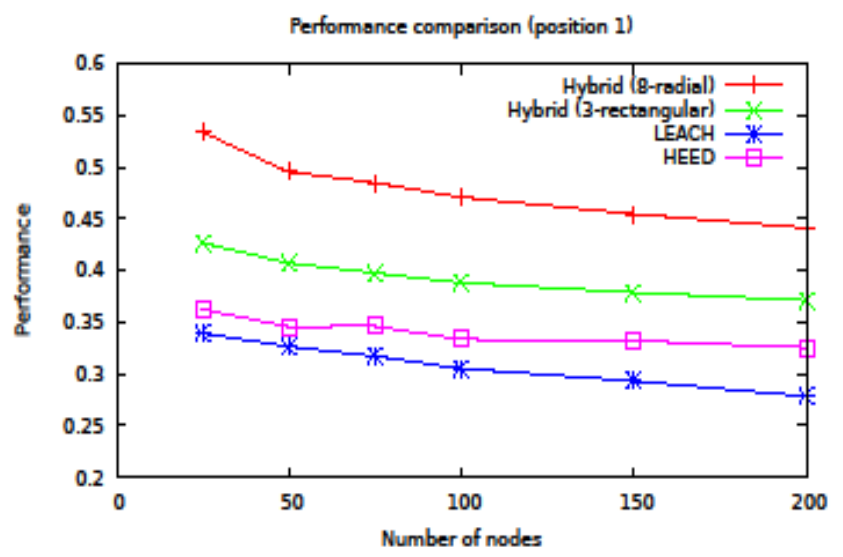

Fig. 8. Energy consumption.

They also show that the greater the number of nodes, the more efficient our algorithm is especially in radial mode. The rectangular mode and radial mode are satisfactory as LEACH and HEED solutions. HEED is more efficient than LEACH in the presence of many nodes. Comparing with our algorithm, we note that our algorithm is much more economical at $30 \%$ than LEACH and $25 \%$ than HEED.

\section{DISCUSSION}

LEACH and its derivatives do not take into account the realities on the ground. The methods are based on events. The amount of remaining energy is the main source in the choice of cluster-heads. The location of the cluster relative to the bore head is ignored. The position of the base station for critical events is usually different from the center of the well field.

However, the energy loss for the nodes waiting for a message from another node with low energy capacity is enormous.

Also, sending erroneous messages due to the low energy level raises a problem of insecurity in the network of sensors. Our algorithm ensures a good life to sensor networks. This algorithm can also extend the life of the network of sensors by putting out unnecessary nodes for the network operation.

Nodes do not die randomly. A metric allows each node in a cluster to die at the right time. This algorithm provides an easy selection of the cluster head, avoiding a lot of communication between the nodes. Each cluster is made of autonomous nodes. 
Experiments on SMAC showed an improvement in the life of the sensor network compared with LEACH and HEED. This method provides a better quality service than LEACH, HEED.

It can be extended to other methods. However, our algorithm is perfectible about choosing the nearest neighbor. Also, it can be improved to update routing tables when a node disappears.

\section{CONCLUSION}

In this paper, we have been able to make a state of the art on the hierarchy in sensor networks to save energy. We proposed a solution to bring an additional quality in management. This quality is ignored in the basic algorithms of clustering and is the main thrust of new research in the coming years. This study has also allowed us to see the limits of the practice of certain solutions.

Our solution allows to propose a maintenance algorithm to avoid a loss of energy caused by a failed node energy. It also saves the energy expended by the formation of clusters and cluster heads.

In our implementation, we have determined the number of effective areas for a large scale of sensor network. Three thresholds have allowed us to evaluate and maintain the sensors for their role in the sensor network.

As prospects, we will try to adapt our algorithm for mobile sensor networks to further reduce the energy of sensors and sensor network energy consumption.

\section{REFERENCES}

[1] K. Beydoun, V. Felea, and H. Guyennet, "Wireless sensor network system helping navigation of the visually impaired," in Proc. 2008 3rd International Conference on Information and Communication Technologies: From Theory to Applications, 2008.

[2] J. Y. Hwang, J. M. Kang, Y. W. Jang, and H. Kim, "Development of novel algorithm and real-time monitoring ambulatory system using Bluetooth module for fall detection in the elderly," in Proc. Annual International Conference of the IEEE Engineering in Medicine and Biology Society, IEEE Engineering in Medicine and Biology Society, 2004.

[3] W. R. Heinzelman, A. Chandrakasan, and H. Balakrishnan, "Energy-efficient communication protocol for wireless microsensor networks," in Proc. the 33rd Annual Hawaii International Conference on System Sciences, 2000.

[4] T. Arici, B. Gedik, Y. Altunbasak, and L. Liu, "PINCO: a pipelined in-network compression scheme for data collection in wireless sensor networks," in Proc. 12th IEEE International Conference on Computer Communications and Networks, 2003, pp. 539-544.

[5] E. P. Capo-Chichi, H. Guyennet, and J.-M. Friedt, "K-RLE: A new data compression algorithm for wireless sensor network," in Proc. 2009 Third International Conference on Sensor Technologies and Applications, IEEE, 2009, pp. 502-507.

[6] J. Higuera, J. Polo, and M. Gasulla, "A Zigbee wireless sensor network compliant with the IEEE1451 standard," in Proc. 2009 IEEE Sensors Applications Symposium, 2009, pp. 309-313.

[7] Y. Li, "Research on application of ZigBee technology in flammable and explosive environment," Wireless Sensor Network, vol. 2, no. 6, pp. 467-471, 2010.

[8] I. Akyildiz, "A survey: Computer networks," Wireless Sensor Networks, vol. 38, no. 4, pp. 393-422, 2002.

[9] A. Manjeshwar and D. P. Agrawal, "TEEN: a routing protocol for enhanced efficiency in wireless sensor networks," in Proc. 15th International Parallel and Distributed Processing Symposium, 2001.
[10] A. Manjeshwar and D. P. Agrawal, "APTEEN: a hybrid protocol for efficient routing and comprehensive information retrieval in wireless," in Proc. 16th International Parallel and Distributed Processing Symposium, 2002.

[11] S. Lindsey and C. S. Raghavendra, "PEGASIS: Power-efficient GAthering in sensor information systems." Comput. Syst. Res. Dept., Aerosp. Corp., Los Angeles, CA, USA, 2002.

[12] M. Ye, C. Li, G. Chen, and J. Wu, "EECS: An energy efficient clustering scheme in wireless sensor networks," in Proc. PCCC 2005. 24th IEEE International Performance, Computing, and Communications Conference, 2005, pp. 535-540.

[13] N. Mejri and F. Kamoun, Algorithme de Routage Hiérarchique MHEED a Plusieurs Sauts pour Les Grands Réseaux de Capteurs, 2010.

[14] L. Dehni and F. Krief, "LEA2C: Une nouvelle approche de routage dans les réseaux de capteurs pour l'optimisation de la consommation d'énergie, " 2005.

[15] W. R. Heinzelman, A. Chandrakasan, and H. Balakrishnan, "An application-specific protocol architecture for wireless microsensor networks," IEEE Transactions on Wireless Communications, vol. 1, no. 4, October 2002.

[16] S. J. Lee, J. S. Lee, H. Sin, S. Yoo, S. Y. Lee, J. Lee et al., "An energy-efficient distributed unequal clustering protocol for wireless sensor networks," Engineering and Technology, pp. 443-447, 2008.

[17] O. Younis and S. Fahmy, "HEED: A hybrid, energy-efficient, distributed clustering approach for ad-hoc sensor networks," Simulation, pp. 1-36, 2004.

[18] P. Duan and H. U. I. Li, "ZigBee wireless sensor network based multi-agent architecture in intelligent inhabited environments," in Proc. 4th International Conference on Intelligent Environments, 2008.

[19] A.-K. Chandra-Sekaran, A. Nwokafor, P. Johansson, K. D. Mueller-Glaser, and I. Krueger, "ZigBee sensor network for patient localization and air temperature monitoring during emergency response to crisis," in Proc. 2008 Second International Conference on Sensor Technologies and Applications, 2008, pp. 233-238.

[20] X. Fan, "Sensors dynamic energy management in WSN," Wireless Sensor Network, vol. 2, no. 9, pp. 698-702, 2010.

[21] R. V. Kulkarni, A. Forster, and G. K. Venayagamoorthy, "Computational intelligence in wireless sensor networks: A survey," IEEE Communications Surveys and Tutorials, vol. 13, no. 1, pp. 68-96, 2011.

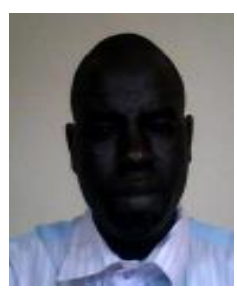

Yacouba Ouattara was born in 1973 at Banfora, Burkina Faso. He is a fourth-year $\mathrm{PhD}$ candidate in computer science at Franche comt'e University. His research interests include distributed systems, security, networks, wireless sensor networks.

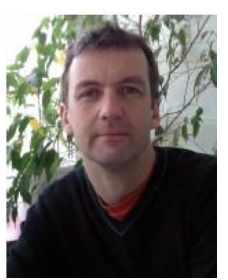

Christophe Lang was born in 1972 at Macon, France. $\mathrm{He}$ got a $\mathrm{PhD}$ degree in computer science from University of Franche-Comt'e, in 1999. He has been an associate professor in computer science in University of Franche-Comt since 2000. His research interests focus on distributed systems, multi-agent systems, simulation, models and sensor networks.

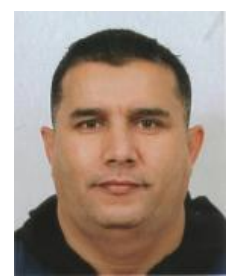

Adel Elgaber is a Ph.D. student in computer science at the Department of Computer Science for Complex Systems in University of Franche-Comt'e, Besanon, France. He is working on wireless sensor networks. 